\title{
Potential Field Cellular Automata Model for Pedestrian Evacuation in a Domain with a Ramp
}

\author{
Xiao-Xia Jian and Xiaoning Zhang \\ School of Economics and Management, Tongji University, Shanghai 200092, China \\ Correspondence should be addressed to Xiaoning Zhang; cexzhang@tongji.edu.cn \\ Received 14 November 2013; Accepted 23 December 2013; Published 11 February 2014 \\ Academic Editor: Hu Shao
}

Copyright (C) 2014 X.-X. Jian and X. Zhang. This is an open access article distributed under the Creative Commons Attribution License, which permits unrestricted use, distribution, and reproduction in any medium, provided the original work is properly cited.

We propose a potential field cellular automata model with a pushing force field to simulate the pedestrian evacuation in a domain with a ramp. We construct a cost potential depending on the ramp angle and introduce a function to evaluate the pushing force, which is related to the cost and the desired direction of pedestrian. With increase of crowd density, there is no empty space for pedestrian moving forward; pedestrian will purposefully push another pedestrian on her or his desired location to arrive the destination quickly. We analyse the relationship between the slope of ramp and the pushing force and investigate the changing of injured situations with the changing of the slope of ramp. When the number of pedestrians and the ramp angle arrive at certain critical points, the Domino effect will be simulated by this proposed model.

\section{Introduction}

Crowd dynamics is the universal phenomenon and also the source of the catastrophe, for example, crowd disaster at the 2010 Love Parade electronic dance music festival in Duisburg, stampede ensued during Beckham's visit to Tongji University in Shanghai on June 20, 2013, and stampedes during largescale sport and entertainment activities. Studying reasons of the catastrophe is quite critical to reduce the occurrence of disasters in the crowd. A plenty of research works, using video analysis [1-3], field study [1,4], and modelling [5-8], focus on investigating the macroscopic crowd dynamics and microscopic variable characteristics exhibited by different individual pedestrians, analysing pedestrian dynamics in various scenarios. One of critical behavioral reactions for pedestrians is the pushing force among crowd under usual situation, which cannot be ignored in exploring the causes of crowd disaster.

As for our knowledge, the usual situation is that each pedestrian who prepares to attend the activities in stadiums or concerts is fully aware that he or she will experience high density crowd before entering the field; hence, his or her motion is stabile and the physical contact is the main factor to produce the pushing force. When people are condensed to some critical density, marginal physical forces of each individual add up to a lethal pressure and this is even true without the panic which inevitably bursts in such occasions. In particular, if the walking domain is even, for instance, a passageway with a ramp, the pushing force is affected by this external condition. In this case, pedestrians hold rational cogitations, so they desire to move with minimal travel cost or travel time, as described in [8] and references therein. In the potential field CA model, the cost potential [8] is the navigation of pedestrian movement, and the pedestrian moves along the direction of minimizing the cost, but this model is inadequate to simulate the pushing force among pedestrians.

A plenty of works focus on describing the avoidance behavior between bidirectional pedestrian flows [5, 7-13], others consider the friction $[11,14,15]$ and repulsion $[11,15]$. However, the contributions on investigating the pushing force $[11,16,17]$ reproduced by physical contacts among pedestrians are scarce. Henein and White [16] introduce a force vector field to simulate the pushing force; Song et al. [17] investigate 
the number of overlapping grids occupied by pedestrians to measure the pushing force. All these works did not discuss the pedestrians evacuation in a domain with the ramp.

In this paper, we describe the pedestrian evacuation in a domain with a ramp. This scenario can be used to simulate the domain around the entrance of stadiums or concert halls. Based on the basic rules of potential field CA model, we construct a new cost potential depending on the ramp angle and introduce a function to evaluate the pushing force, which is related to the cost and the desired direction of pedestrian; in other word, with increase of crowd density, there is no empty space for pedestrian moving forward; pedestrian will purposefully push another pedestrian on her or his desired location to reach the destination quickly; the magnitude of push force is determined by her or his potential value of current location. The former who is close to the exit produces small force and bears maximal pushing forces and the latter produces large force, and bear minimal pushing forces; when the pushing force is beyond a certain critical value, the pedestrian will fall onto the ground. What is more, the slope of a ramp is an important factor to affect pedestrian evacuation dynamics. When the slope of a ramp is deep, the critical value of pushing force is small and much more pedestrians could be injured; when the slope of ramp is small, the critical value of pushing force is large and fewer pedestrians may experience injury; the value of critical value is maximal when the angle of ramp is zero. When the number of pedestrians and the ramp angle are beyond certain critical points, the Domino effect will be simulated by this proposed model.

The remainder of this paper is organized as follows. In Section 2, we formulate a potential field cellular automata model with a pushing force field. In Section 3, the process of pedestrian evacuation in a domain with a ramp is simulated and the injured phenomenon that resulted from the pushing influences is reproduced to verify the practicality of this model; What is more, we analyse the sources of pushing disasters; one is the pushing force produced by local pedestrian group, which is dependent on the potential field; another is the deep ramp. Section 4 concludes the paper.

\section{Potential Field CA Model with Pushing Force in Ramp}

In this paper, we investigate the pedestrian evacuation in the walking domain with a ramp, where the walking domain is represented by $\widehat{\Omega}$, and the scenario is shown in Figure 1. We simulate pedestrians going across subdomain $C, \operatorname{ramp} B$, and subdomain $A$ and then leaving the walking domain from the exit at left side of subdomain $A$. Initially, pedestrians are assigned randomly to subdomains $B$ and $C$ with the initial density $\rho_{0}$.

The walking domain $\widehat{\Omega}$ is divided into cells with the space step $h$ along $x$ direction ( $y$ direction); each cell is empty or occupied by at most one pedestrian. After determining his or her movement direction, each pedestrian moves into his desired direction. The current position and possible movement direction of each pedestrian are shown in Figure 2(a).

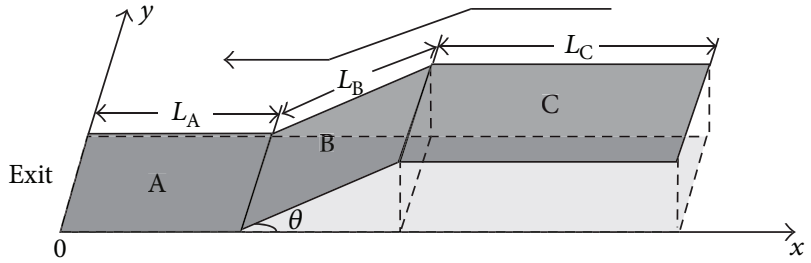

FIGURE 1: Illustration of the walking domain with a ramp. The walking domain $\widehat{\Omega}$ (gray) is constructed by three subdomains $A, B$, and $C$; level subdomains $A$ and $C$ are connected by ramp $B$. Here, $L_{A}, L_{B}$, and $L_{C}$ are the lengths of subdomains, respectively; the black arrow indicates the direction of motion.

Here we assume that $h=0.4 \mathrm{~m}$ which produces an average area occupied by one pedestrian [10]; therefore, the dimensionless maximal density $\rho_{\max }=1$ corresponds to actual density $6.25 \mathrm{ped} / \mathrm{m}^{2}$. Besides, as the average density of real pedestrian is $1 \mathrm{~m} / \mathrm{s}$, we assume that the maximum speed of each pedestrian in our model is $v_{\max }=1 \mathrm{~m} / \mathrm{s}$ and it implies that the time step is $0.4 \mathrm{~s}$. The crucial step of the CA model is to determine the transition probabilities of Figure 2(b). In this proposed model, the transition probabilities are based on a potential field and a pushing force field.

2.1. Potential Field in a Domain with a Ramp. The potential field is a navigation of the pedestrian walking; here, the walking domain $\widehat{\Omega}$ is not on a two-dimensional plane. In order to compute potential field in the uneven walking domain $\widehat{\Omega}$, firstly, using $(x, y)$ to represent the coordinate of the cell, we compute the virtual potential $\phi(x, y, t)$ on two-dimensional level domain $\Omega$ with the exit $\Gamma_{0}$ at each time $t . \Omega$ is set by leveling down $\widehat{\Omega}$; secondly, we reconstruct the potential $\phi(x, y, t)$ by introducing a ramp coefficient $\omega(x, y, \theta)$. The mathematical formulations of calculating the potential field $\widehat{\phi}(x, y, t, \theta)$ in $\widehat{\Omega}$ are as follows.

(a) The virtual potential $\phi(x, y, t)$ is determined by solving the following Eikonal equation:

$$
\begin{gathered}
\|\nabla \phi(x, y, t)\|=\tau(x, y, t), \quad(x, y) \in \Omega, \\
\phi\left(x_{0}, y_{0}, t\right)=0, \quad\left(x_{0}, y_{0}\right) \in \Gamma_{0},
\end{gathered}
$$

where $\|\nabla \phi(x, y, t)\|=\left(\phi_{x}(x, y, t)^{2}+\phi_{y}(x, y, t)^{2}\right)^{1 / 2}$ and $\tau(x, y, t)$ is a cost distribution function which satisfies that $\tau(0)=1, \tau^{\prime}(\rho)>0,0 \leq \rho \leq 1[8,12$, 13]. The existence and uniqueness of the solution of problem (1) are discussed by [8], and this problem can be solved by using the fast sweeping method [18].

(b) The potential $\widehat{\phi}(x, y, t, \theta)$ is reconstructed by $\phi(x, y, t)$ multiplying a ramp coefficient $\omega(x, y, \theta)$; that is,

$$
\widehat{\phi}(x, y, t, \theta)=\omega(x, y, \theta) \phi(x, y, t),
$$




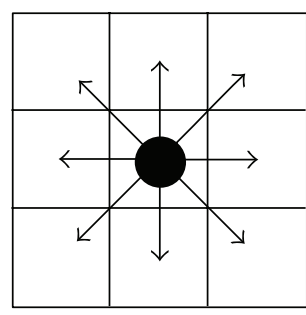

(a)

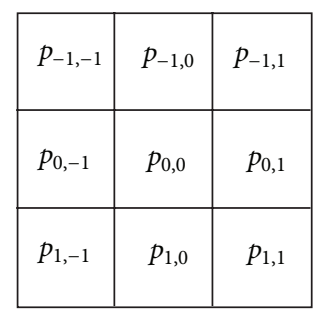

(b)

Figure 2: (a) An occupied cell and its eight neighboring cells, corresponding to (b) the nine probabilities for the pedestrian in the occupied cell to update his or her position.

where

$$
\begin{aligned}
& \omega(x, y, \theta) \\
& =\left\{\begin{array}{l}
1, \quad x \in\left[0, L_{A}\right], \\
\exp \left(\alpha \frac{x-L_{A}}{h} \tan \theta\right), \\
\quad x \in\left(L_{A}, L_{A}+L_{B} \cos \theta\right), \\
\exp \left(\alpha \frac{L_{B}}{h} \sin \theta\right), \\
\quad x \in\left[L_{A}+L_{B} \cos \theta, L_{A}+L_{B} \cos \theta+L_{C}\right] .
\end{array}\right.
\end{aligned}
$$

$\alpha$ is a parameter that reflects the sensitivity of potential to the slope of ramp.

Obviously, we magnify the potential $\phi(x, y, t)$ by the coefficient $\omega(x, y, \theta) \geq 1$, which suggests a stronger effect with a deeper ramp angle; in other words, $\omega(x, y, \theta)$ is increasing with the ramp angle $\theta \in\left[0,90^{\circ}\right)$. Here, after giving an angle $\theta$ of the ramp, the weight function $\omega(x, y, \theta)$ is given; although subdomains $A$ and $C$ are all flat, the height difference between two flat subdomains $A$ and $C$ is different with different $\theta$; thus, the values of weight $\omega(x, y, \theta)$ in subdomain $A$ are different than the ones in subdomain $C$, which implies that the potential values in subdomain $C$ are influenced by the ramp angle $\theta$. In addition, the final potential $\widehat{\phi}(x, y, t, \theta)$ is consistent with $\phi(x, y, t)$ if $\theta=0$.

2.2. Aggregated Pushing Force Field. In the crowd, each pedestrian is surrounded by other pedestrians, hence, physical contacts among pedestrians occur, and pedestrians produce the pushing forces to desire for increasing the personal freespace and being close to the destinations. In the proposed model, we introduce the aggregated pushing force field to simulate the physical force effects.

While the density $\rho(x, y)$ is equal to the maximal density 1 $\left(6.25 \mathrm{ped} / \mathrm{m}^{2}\right)$, which means that there is no empty neighbour cell for the pedestrian in $(x, y)$ to arrive, the magnitude of the pushing force produced by the pedestrian in $(x, y)$ at time $t$ is

$$
f(x, y, t)=\left(1-\frac{\widehat{\phi}(x, y, t, \theta)}{\max _{(\widehat{x}, \widehat{y}) \in \widehat{\Omega}} \widehat{\phi}(\widehat{x}, \widehat{y}, t, \theta)}\right)^{4} .
$$

This functional $f(x, y, t)$ reflects the sensitivity of pushing force to potential field, which is not a real pushing force. The functional $f(x, y, t)$ implies that the pushing force is decreasing with potential $\widehat{\phi}(x, y, t, \theta)$. In practice, while the pedestrian is near to the exit, his or her potential is much smaller than the maximal potential of all the domain and he or she holds stronger propulsion; on the contrary, the distance between the pedestrian's location and the exit is large; he or she is patient with small pushing force. The pedestrian considers that the pushing is helpful in arriving at the destination quickly; thus, he or she pushes along the direction of minimizing the cost potential $\widehat{\phi}(x, y, t, \theta)$; that is, the direction of $\vec{f}(x, y, t)$ is $\vec{l}(x, y, t)=\left(l_{1}, l_{2}\right)=-\left(\widehat{\phi}_{x}, \widehat{\phi}_{y}\right) /\|\nabla \widehat{\phi}\|$.

In fact, the pedestrian's pushing force is repeatedly retransmitted from person to person through interpersonal contacts within crowds [19]. In this proposed model, we model this process by adding up the pushing force in each time step. The incremental pushing force $\vec{f}^{\text {add }}$ in each cell $(x, y)$ at each time step is produced by whether this pedestrian in $(x, y)$ is directed by another pedestrian's desired direction. Here, using $\vec{f}^{\text {add }}(x, y, t)$ to represent the pushing force experienced by pedestrian in $(x, y)$, if there exists a pedestrian in $(x, y)$ 's neighbour $(\bar{x}, \bar{y})$ whose desired walking direction $\left(l_{1}, l_{2}\right)=(x-\bar{x}, y-\bar{y})$, pushing force $\vec{f}(\bar{x}, \bar{y}, t)$ is produced by pedestrian in $(\bar{x}, \bar{y})$ and experienced by the pedestrian in $(x, y)$, where the magnitude $f^{\text {add }}(x, y, t)$ is equal to $f(\bar{x}, \bar{y}, t)$ and the direction is also along $\left(l_{1}, l_{2}\right)$; otherwise, $\vec{f}^{\text {add }}=0$.

Therefore, the aggregated pushing force (vector-wise) experienced by the pedestrian in the cell $(x, y)$ is the vector sum of forces generated by the aggregated force from the beginning to time step $t-1$ and the incremental pushing force $\vec{f}^{\text {add }}(x, y, t)$. Obviously, $f^{\text {add }}(x, y, t) \geq 0$. Using function (4), we define the aggregated force vector field $\vec{F}(x, y, t)$ experienced by each pedestrian in $(x, y)$ as

$$
\begin{aligned}
\vec{F}(x, y, t) & =\vec{F}(x, y, t-1)+f^{\text {add }}(\bar{x}, \bar{y}, t) \cdot \vec{l} \\
& =\left(F^{1}(x, y, t), F^{2}(x, y, t)\right),
\end{aligned}
$$

where $F^{1}(x, y, t)$ and $F^{2}(x, y, t)$ are two components of $\vec{F}(x$, $y, t)$ along axes $x$ and $y$, respectively. For convenience, $\|\vec{F}(x, y, t)\|=\left(\left(F^{1}(x, y, t)\right)^{2}+\left(F^{2}(x, y, t)\right)^{2}\right)^{1 / 2}$ represents the magnitude of $\vec{F}(x, y, t)$. 
If $\|\vec{F}(x, y, t)\|$ exceeds a threshold, the pedestrian will become injured, and the injured pedestrians could not move again. Here, the threshold parameter, represented by $F^{*}(x, y, t)$, is the increasing function of pushing force $f(x$, $y, t)$ and is dependent on the angle of ramp. The mathematical formulation of $F^{*}(x, y, t)$ is defined as

$$
F^{*}(x, y, t)=\left\{\begin{array}{l}
\beta \cdot \frac{f(x, y, t)}{\cos \theta}, \\
x \in\left(L_{A}, L_{A}+L_{B} \cos \theta\right), \\
\beta \cdot f(x, y, t), \\
x \in\left[0, L_{A}\right] \cup\left[L_{A}+L_{B} \cos \theta,\right. \\
\left.L_{A}+L_{B} \cos \theta+L_{C}\right],
\end{array}\right.
$$

where $\beta$ is a sensibility parameter and the formulation of $\beta$ is defined as

$$
\beta= \begin{cases}10, & \theta=0, \\ \csc \theta, & \theta \in\left(0,90^{\circ}\right) .\end{cases}
$$

Here, $\beta$ is a decreasing function related to the angle of ramp. While $\theta$ is zero, $\beta$ is a large value and it implies that the pedestrian in this situation is not easily pushed onto fall the ground; with increase of $\theta, \beta$ decreases and it implies that the pedestrian is gradually easily pushed down. Obviously, another choice of critical threshold can be used to deeply investigate the different pushing processes of crowd dynamics.

2.3. Update Rules. The cost potential field $\widehat{\phi}(x, y, t, \theta)$ is calculated using (1) and (2) with current density distribution $\rho(x, y, t)$. The force field $\vec{F}(x, y, t)$ is calculated by formula (5). In this section, the transition probabilities and the injured probabilities are bounded by the comparisons between the $\|\vec{F}(x, y, t)\|$ and $F^{*}(x, y, t)$. Assign $(0,0)$ to cell occupied by uninjured pedestrian and then the update rules from time step $t$ to $t+1$ are as follows.

(1) If $\|F(x, y, t)\| \leq F^{*}(x, y, t)$, the pedestrian moves by the transition probabilities $p_{i, j}$ shown in Figure 2(b).

(1.1) $p_{i, j}$ is determined by

$$
\begin{aligned}
& p_{i j}= \begin{cases}\frac{1}{\left|S_{m}\right|}, & (i, j) \in S_{m}, \\
0, & (i, j) \notin S_{m},\end{cases} \\
& \text { if } S_{m} \neq \emptyset, \delta \widehat{\phi}_{\min }(0)<0 ;
\end{aligned}
$$

otherwise

$$
p_{i j}= \begin{cases}1, & (i, j)=(0,0), \\ 0, & (i, j) \neq(0,0),\end{cases}
$$

where $\delta \widehat{\phi}_{i j}(0) \equiv(\widehat{\phi}(i, j)-\widehat{\phi}(0,0)) / \iota_{i j}$ for $(i, j) \epsilon$ $S_{0}$ is the difference quotient, with $S_{0}=\{(i, j) \mid$ $(i, j)$ is empty\}, and $\iota_{i j}$ is the distance between the cells $(i, j)$ and $(0,0)$. Besides, the set
$S_{m}=\left\{(i, j) \mid \delta \widehat{\phi}_{i j}(0)=\delta \widehat{\phi}_{\min }(0)\right\} \subseteq S_{0}$, where

$\delta \widehat{\phi}_{\min }(0)=\min _{(i, j) \in S_{0}} \delta \widehat{\phi}_{i j}(0)$, and $\left|S_{m}\right|$ is the number of elements in $S_{m}$.

(1.2) Under the parallel update, conflicts that resulted from the fact that an empty cell $(i, j)$ could be a target cell of $m(0 \leq m \leq 8)$ pedestrians in the $m$ neighboring cells $\left(0^{(k)}, 0^{(k)}\right)$ if $m \geq 2$ can be resolved by the following probabilities:

$$
p\left(0^{(k)}\right)=\left\{\begin{array}{l}
\frac{1}{\left|S_{m m}\right|}, \\
\text { if } m \geq 1,\left(0^{(k)}, 0^{(k)}\right) \in S_{m m}, \\
0, \quad \\
\text { otherwise, }
\end{array}\right.
$$

where

$$
\begin{gathered}
S_{m m}=\left\{\left(0^{(k)}, 0^{(k)}\right) \mid \delta \widehat{\phi}_{\min }\left(0^{(k)}\right)=\delta \widehat{\phi}_{\min }, 1 \leq k \leq m\right\}, \\
\delta \widehat{\phi}_{\min }=\min _{1 \leq k \leq m} \Delta \widehat{\phi}_{\min }\left(0^{(k)}\right) .
\end{gathered}
$$

(2) If $\|F(x, y, t)\|>F^{*}(x, y, t)$, the pedestrian will be injured by the probabilities $\bar{p}_{i, j}$.

(2.1) The injured probabilities are defined by

$$
\bar{p}_{i, j}=r^{\text {injured }} p_{i, j}
$$

where $r^{\text {injured }} \in[0,0.5]$ is a random parameter. In this case, the pedestrian in $(0,0)$ is injured and falls in the location $(i, j)$ with the probabilities $\bar{p}_{i, j}$; when $(i, j) \neq(0,0)$, this pedestrian will fall in an empty cell along the direction of his or her aggregated pushing force; otherwise, this pedestrian will fall in the current location.

(2.2) In this case, to avoid the situation that different neighbor pedestrians fall into the same empty cell simultaneously, we set equal fallingin possibility to different neighbor pedestrians; namely,

$$
p\left(0^{(k)}\right)= \begin{cases}\frac{1}{m}, & \text { if } m \geq 1 \\ 0, & \text { otherwise }\end{cases}
$$

One of injured pedestrians falls in the desired cell, and others fall in the current locations.

\section{Simulation}

In this simulation, the sizes of walking domain are $5 \times 3$ cells for subdomain $A, 5 \times 4$ cells for $\operatorname{ramp} B$, and $5 \times 20$ for subdomain $C$. The cost distribution $\tau(x, y, t)$ is given by

$$
\tau(x, y, t)=1+g_{0} \rho^{\gamma}(x, y, t),
$$

where we set $g_{0}=0.075$ and $\gamma=2$. 


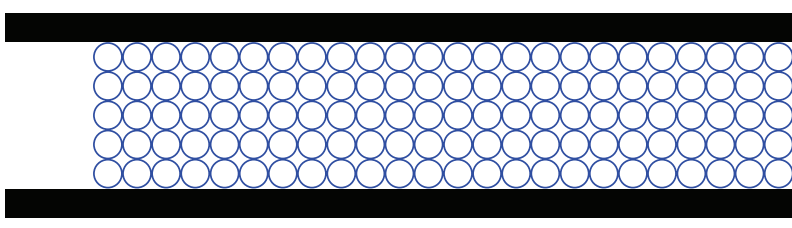

(a)

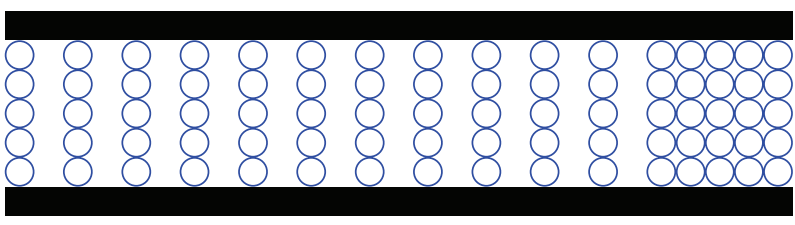

(c)

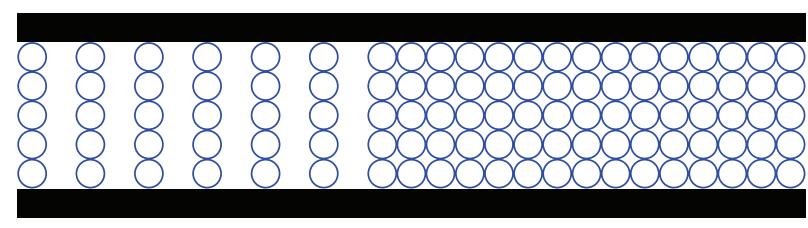

(b)

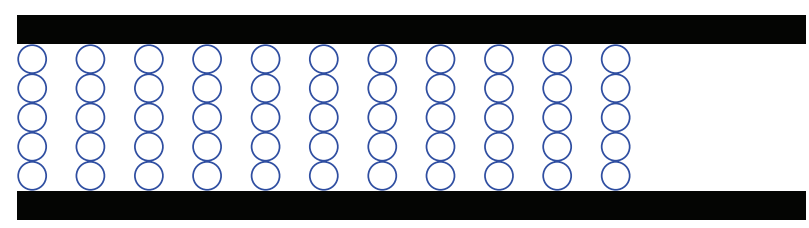

(d)

FIGURE 3: Evacuation process simulated by the proposed model, at $t=0,10,20$, and 30 (color online). The initial density $\rho_{0}=1$ and the angle of $\operatorname{ramp} \theta=0$. The open circles represent pedestrians.

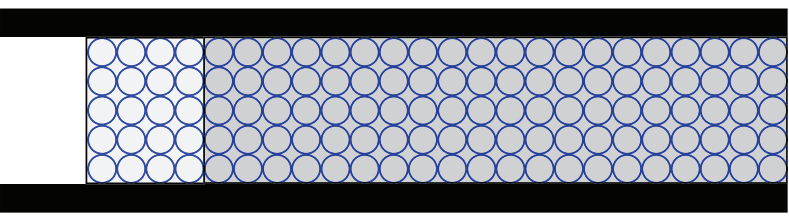

(a)

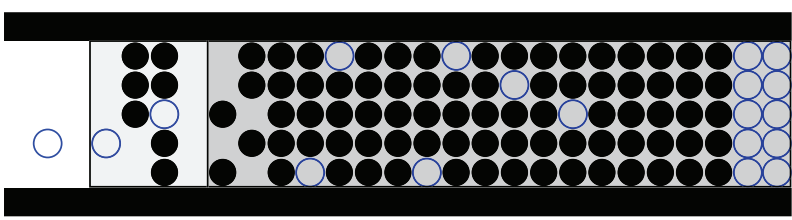

(c)

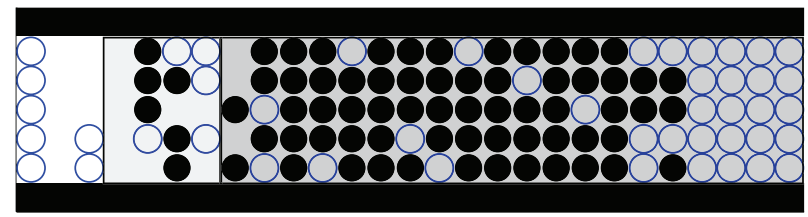

(b)

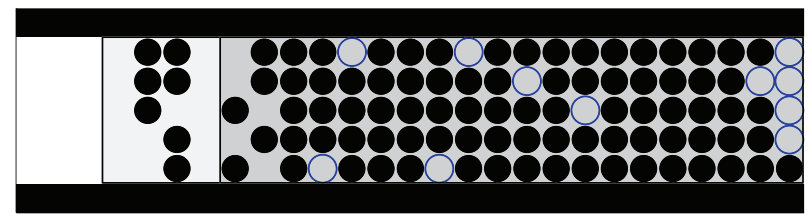

(d)

FIGURE 4: Evacuation process simulated by the proposed model, at $t=0,6,15$, and 30 (color online). The initial density $\rho_{0}=1$ and the angle of $\operatorname{ramp} \theta=50^{\circ}$. Here, the light gray domain represents the projection of the ramp (subdomain $B$ ), and the gray is the subdomain $C$; the normal pedestrians and the injured pedestrians are indicated by open circles and the full circles, respectively.

Initially, $N$ pedestrians $(N<5 \times 4+5 \times 20)$ are randomly assigned to the inner cell in subdomains $B$ and $C$ with the initial mean density $\rho_{0}=N /(5 \times 4+5 \times 20)$. In the simulations, we simulate the injured phenomena and investigate relationships between the angle of the ramp and the crowd falling onto the ground under different initial densities $\rho_{0}$. Figure 3 shows the evacuation process simulated by the proposed model. In this simulation, the walking domain is without the ramp. Figures $3(\mathrm{a})-3(\mathrm{~d})$ are the snapshots of an evacuation simulation at $t=0,10,20$, and 30, respectively. This is in accordance with the underlying principle that the path-choice strategy in the propose model is somewhat optimal due to the pedestrians' awareness of the destination and the surroundings.

The other simulation is to investigate the evacuation dynamics in the walking domain with the ramp. Figure 4 shows the overlooked evacuation process in this situation here, angle of the ramp is $\theta=50^{\circ}$. Obviously, Figures 4(b)$4(\mathrm{~d})$ show the injured phenomena; several pedestrians were injured by aggregated pushing force. In addition, the pushing force can result in the Domino effect; from Figure 4(a) to Figure 4(b), after the front pedestrian falls onto the ground, the latter lacking the brace to fall down resulted from the large pushing force. And then, the injured numbers are not significantly increased from Figure 4(c) to Figure 4(d).

The functional relationship between the injured numbers and the angle of the ramp is shown in Figures 5(a) and 5(b), which are simulated by the proposed model. Figures 5(a) and 5 (b) correspond the low initial density $\left(\rho_{0} \in(0,0.5)\right)$ and the high initial density $\left(\rho_{0} \in(0.5,1]\right)$, respectively. According to these curves, the injured numbers increase as the angle of the ramp increases. Moreover, one curve is always above the other, which corresponds to a higher average density, implying that the injured numbers increases with $\rho_{0}$ for a fixed ramp. These results concur with common sense, especially when the initial average density $\rho_{0}$ is increasing, which should give rise to many more pushing effects. Obviously, the curves in Figure 5(a) increase continuously as the angle of the ramp increases; with the high initial density, that is, $\rho_{0}>0.5$, while 


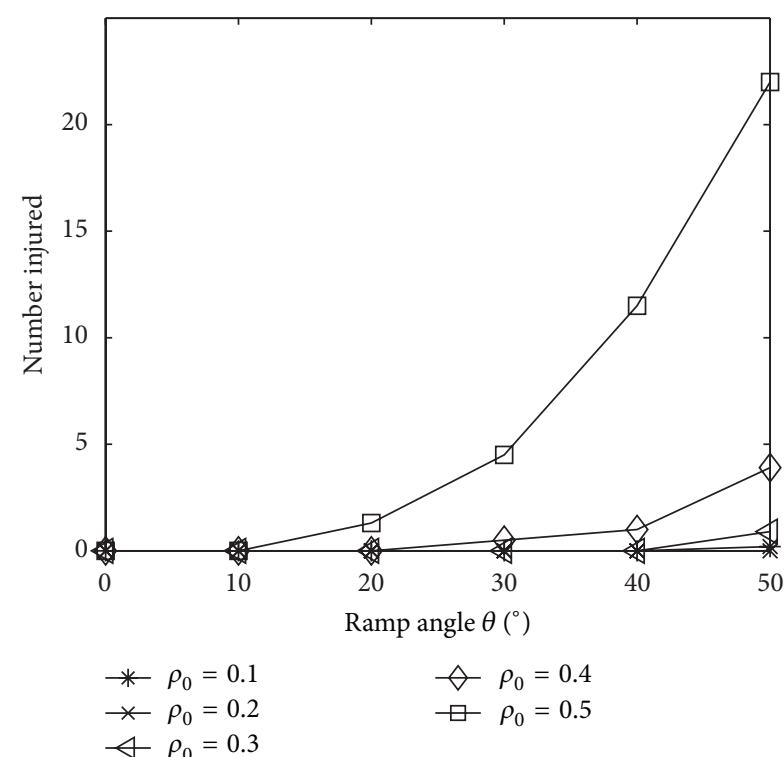

(a)

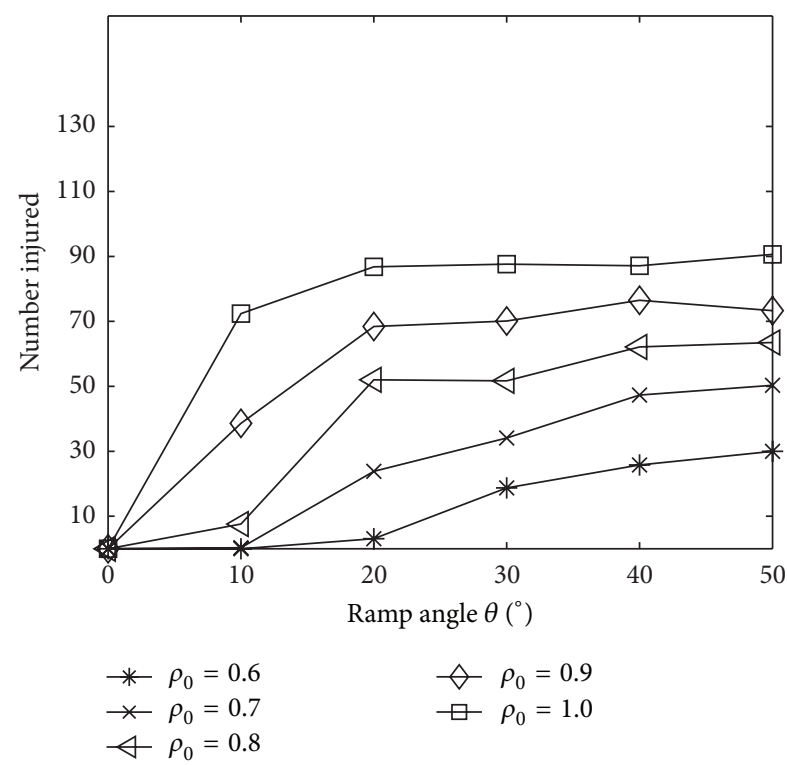

(b)

FIGURE 5: Functional relations between the injured number and the angle of the ramp for a variety of the average densities $\rho_{0}$, simulated by the proposed model. (a) $\rho_{0} \leq 0.5$; (b) $\rho_{0}>0.5$. For each data, an average over 10 runs is plotted.

the ramp is more than $20^{\circ}$, the curves in Figure $5(\mathrm{~b})$ reach a critical value and then the injured number will increase slowly and it implies that the Domino effect is obtained.

\section{Conclusion}

In this paper, a potential field CA model with an aggregated force field is proposed to reproduce pedestrian evacuation in a walking domain with ramp exit. And A potential field is defined to navigate pedestrian movement along minimizing the travel cost paths. In addition, an aggregated pushing force vector field is introduced to simulate the pushing force among pedestrians when pedestrian cannot move forward in high density domain. We investigate the effects of both the aggregated pushing force for pedestrian evacuation and the slope of ramp exit, the conclusion is that these two factors are the key causes of disasters. We will adjust the model parameters according to plentiful experiments and field studies in future work and will jointly simulate the pedestrian dynamics quantitatively and qualitatively using the proposed model.

\section{Conflict of Interests}

The authors declare that there is no conflict of interests regarding the publication of this paper.

\section{Acknowledgment}

This study was supported by the National Natural Science Foundation of China (Grant no. 71125004).

\section{References}

[1] D. Helbing, A. Johansson, and H. Z. Al-Abideen, "Dynamics of crowd disasters: an empirical study," Physical Review E, vol. 75, no. 4, Article ID 046109, 2007.

[2] D. Helbing and P. Mukerji, "Crowd disasters as systemic failures: analysis of the love parade disaster," EPJ Data Science, vol. 1, no. 1, pp. 1-40, 2012.

[3] B. Krausz and C. Bauckhage, "Loveparade 2010: automatic video analysis of a crowd disaster," Computer Vision and Image Understanding, vol. 116, no. 3, pp. 307-319, 2012.

[4] S. C. Wong, W. L. Leung, S. H. Chan et al., "Bidirectional pedestrian stream model with oblique intersecting angle," Journal of Transportation Engineering, vol. 136, no. 3, pp. 234-242, 2010.

[5] H. Kuang, X. L. Li, T. Song, and S. Q. Dai, "Analysis of pedestrian dynamics in counter flow via an extended lattice gas model," Physical Review E, vol. 78, no. 6, Article ID 066117, 2008.

[6] H. Kuang, X. L. Li, Y. F. Wei, T. Song, and S. Q. Dai, "Effect of following strength on pedestrian counter flow," Chinese Physics B, vol. 19, no. 7, Article ID 070517, 2010.

[7] T. Xiong, P. Zhang, S. C. Wong, C. W. Shu, and M. P. Zhang, "A macroscopic approach to the lane formation phenomenon in pedestrian counterflow," Chinese Physics Letters, vol. 28, no. 10, Article ID 108901, 2011.

[8] P. Zhang, X. X. Jian, S. C. Wong, and K. Choi, "Potential field cellular automata model for pedestrian flow," Physical Review E, vol. 85, no. 2, Article ID 021119, 2012.

[9] D. Helbing and P. Molnár, "Social force model for pedestrian dynamics," Physical Review E, vol. 51, no. 5, pp. 4282-4286, 1995.

[10] C. Burstedde, K. Klauck, A. Schadschneider, and J. Zittartz, "Simulation of pedestrian dynamics using a two-dimensional cellular automaton," Physica A, vol. 295, no. 3-4, pp. 507-525, 2001. 
[11] D. Helbing, "Traffic and related self-driven many-particle systems," Reviews of Modern Physics, vol. 73, no. 4, pp. 1067-1141, 2001.

[12] L. Huang, S. C. Wong, M. P. Zhang, C. W. Shu, and W. H. K. Lam, "Revisiting Hughes' dynamic continuum model for pedestrian flow and the development of an efficient solution algorithm," Transportation Research B, vol. 43, no. 1, pp. 127-141, 2009.

[13] Y. Q. Jiang, P. Zhang, S. C. Wong, and R. X. Liu, "A higher-order macroscopic model for pedestrian flows," Physica A, vol. 389, no. 21, pp. 4623-4635, 2010.

[14] A. Kirchner, K. Nishinari, and A. Schadschneider, "Friction effects and clogging in a cellular automaton model for pedestrian dynamics," Physical Review E, vol. 67, no. 5, Article ID 056122, 2003.

[15] W. G. Song, Y. F. Yu, W. C. Fan, and H. P. Zhang, "A cellular automata evacuation model considering friction and repulsion," Science in China E, vol. 48, no. 4, pp. 403-413, 2005.

[16] C. M. Henein and T. White, "Microscopic information processing and communication in crowd dynamics," Physica A, vol. 389, no. 21, pp. 4636-4653, 2010.

[17] W. Song, X. Xu, B.-H. Wang, and S. Ni, "Simulation of evacuation processes using a multi-grid model for pedestrian dynamics," Physica A, vol. 363, no. 2, pp. 492-500, 2006.

[18] H.-K. Zhao, "A fast sweeping method for Eikonal equations," Mathematics of Computation, vol. 74, no. 250, pp. 603-627, 2005.

[19] J. Fruin, "The causes and prevention of crowd disasters," in Engineering for Crowd Safety, R. A. Smith and J. F. Dickie, Eds., Elsevier, Amsterdam, The Netherlands, 1993. 


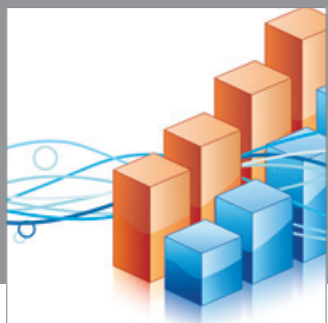

Advances in

Operations Research

mansans

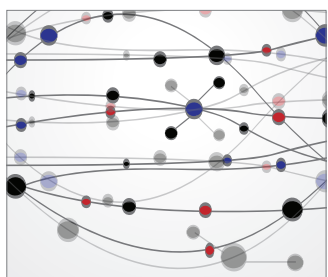

The Scientific World Journal
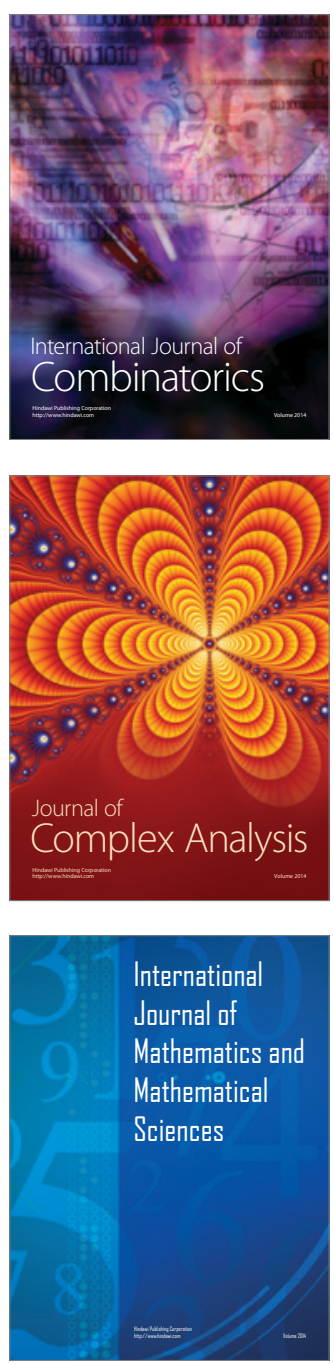
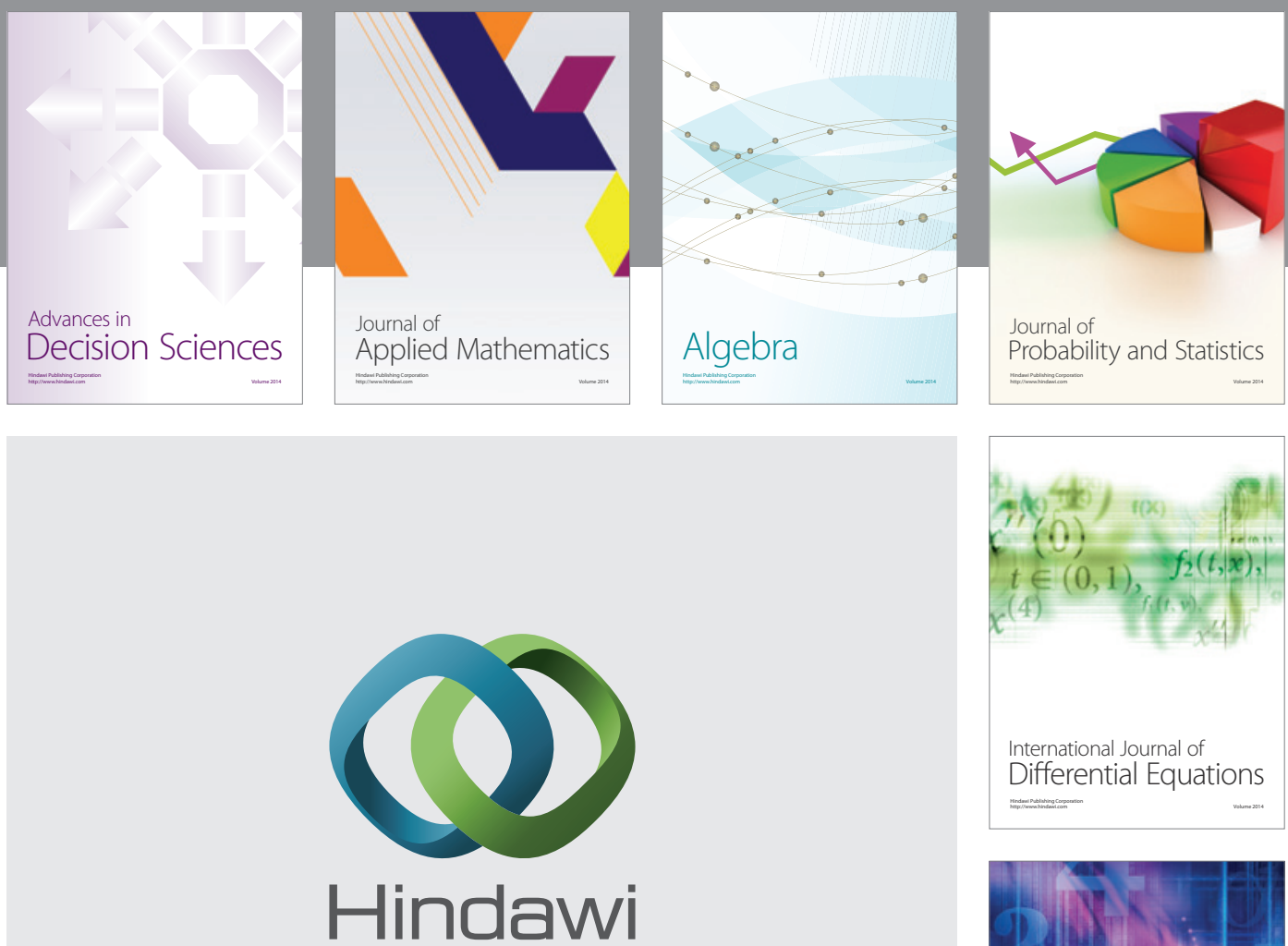

Submit your manuscripts at http://www.hindawi.com
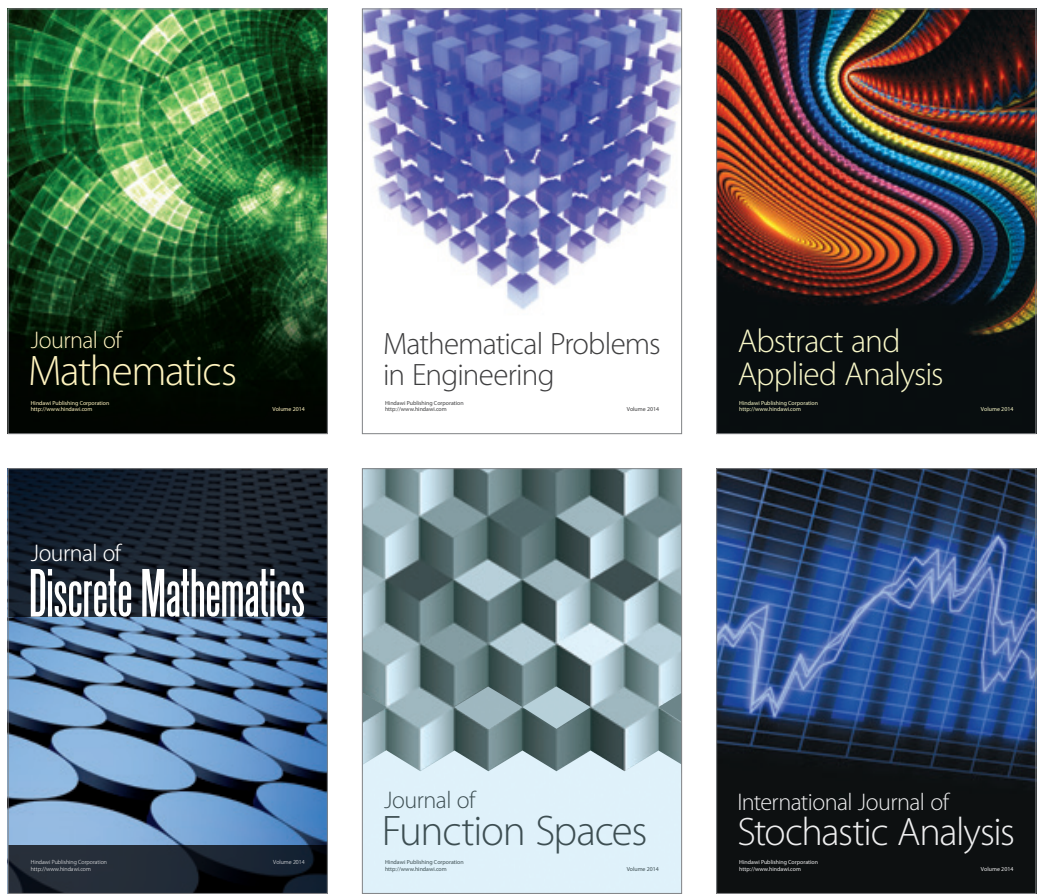

Journal of

Function Spaces

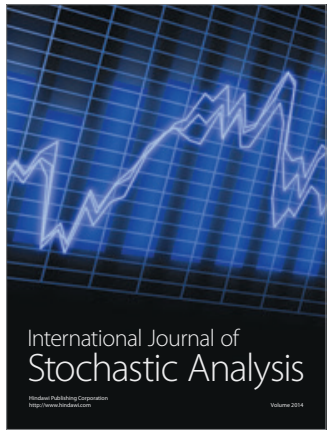

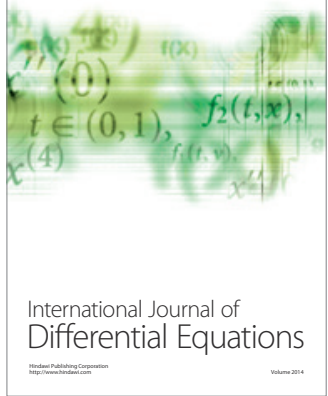
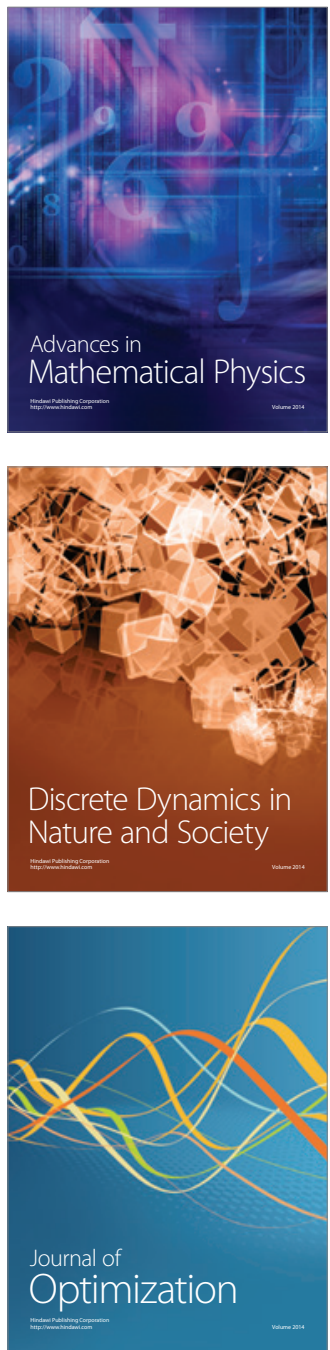